\title{
Le rôle de la SNCF dans le mouvement de la
}

\section{normalisation}

François Lacôte

\section{OpenEdition}

\section{Journals}

Édition électronique

URL : https://journals.openedition.org/rhcf/1954

DOI : 10.4000/rhcf.1954

\section{Éditeur}

Rails \& histoire

\section{Édition imprimée}

Date de publication : 1 février 2003

Pagination : 124-125

ISBN : 0996-9403

ISSN : 0996-9403

\section{Référence électronique}

François Lacôte, "Le rôle de la SNCF dans le mouvement de la normalisation », Revue d'histoire des chemins de fer [En ligne], 26 | 2003, mis en ligne le 20 janvier 2015, consulté le 22 avril 2022. URL http://journals.openedition.org/rhcf/1954 ; DOI : https://doi.org/10.4000/rhcf.1954 


\section{Le rôle de la SNCF dans le mouvement de la normalisation}

Pour les chemins de fer, la normalisation au sens statutaire est apparue relativement tardivement dans toute son ampleur. En effet, compte tenu des contraintes nationales des entreprises publiques, le besoin d'harmonisation s'est fait sentir au départ différemment selon que l'on regarde les stricts besoins des marchés de matériels ou bien ceux de l'interopérabilité.

Dans le premier cas, le quasi-monopole constitué par la SNCF a fait que le besoin de normalisation était interne à l'entreprise et c'est pourquoi celle-ci s'est dotée, dès sa constitution en 1937, d'un organe d'unification technique (standardisation) entièrement tourné vers la préparation de documents internes (ancêtre du Bureau de normalisation et de certification ferroviaires).

Dans le second cas, l'interopérabilité entre réseaux européens, essentiellement pour répondre aux besoins de circulation du matériel remorqué, a entraîné la nécessité d'élaborer des règles techniques adaptées, ce qui constitue la mission de l'Union internationale des chemins de fer.

Aujourd'hui, cette dernière notion, adaptée à la grande vitesse voire généralisée, n’est pas moins d'actualité et elle constitue un des piliers du développement des chemins de fer.

La normalisation utilisable dans les marchés a beaucoup évolué depuis ses débuts, à la fois en raison de la réforme de la normalisation en France (1984), de l'arrivée d'autres acteurs aux côtés de la SNCF et de l'ouverture progressive des marchés.

Le Bureau de normalisation et de certification ferroviaires a ainsi été reconnu par les pouvoirs publics en 1984 avec pour champ de compétence l'ensemble des produits des matériels roulants et des installations fixes, et pour mission de réaliser, au nom de l'Association française de normalisation, les normes nationales ferroviaires (classe $\mathrm{F}$ : 700 normes) et non plus des documents purement internes à la SNCF.

Le Bureau de normalisation et de certification ferroviaires était également chargé de l'interface SNCF/Association française de normalisation pour toutes les normes non ferroviaires (voire non techniques ou organisationnelles) ayant un impact sur la SNCF, et a donc longtemps gêné l'adhésion de la SNCF à l'Association française de normalisation (AFNOR). 
Le Bureau de normalisation et de certification ferroviaires était alors encore financé uniquement par la SNCF malgré son fonctionnement complètement ouvert, mais le pas ultime a été franchi en 1994 par sa transformation en association Bureau de normalisation ferroviaire regroupant de manière équilibrée les exploitants et les industriels. Les mission internes à la SNCF ont été reprises par un service créé à cet effet (NORHA) qui est chargé de la coordination des actions de l'entreprise en ce qui concerne l'ensemble de la normalisation.

Sous la pression des directives régissant les marchés ou l'interopérabilité, le travail de normalisation s'est maintenant déporté vers l'Europe (CEN/CENELEC, Comité européen de normalisation/ Comité européen de normalisation électrotechnique) voire le monde (ISO/CEI, Organisation internationale de normalisation/Commission électrotechnique internationale), et le Bureau de normalisation ferroviaire est devenu progressivement un acteur de la politique de normalisation faisant valoir les intérêts des partenaires français, plutôt qu'un rédacteur de normes. Il exerce son rôle envers le Comité européen de normalisation (CEN) avec une entière délégation de l'Association française de normalisation (AFNOR) et envers le Comité européen de normalisation électrotechnique par une coopération avec l'Union technique de l'électricité (UTE), organisme chargé en général de la représentation française au Comité européen de normalisation électrotechnique.

Aujourd'hui, la normalisation est bien assimilée dans le domaine de la technique d'ingénieur. Mais le développement de la normalisation portant sur des concepts horizontaux (qualité, environnement...) et sur les services amène la SNCF à de nouveaux engagements, y compris dans le domaine de la qualité du transport. 